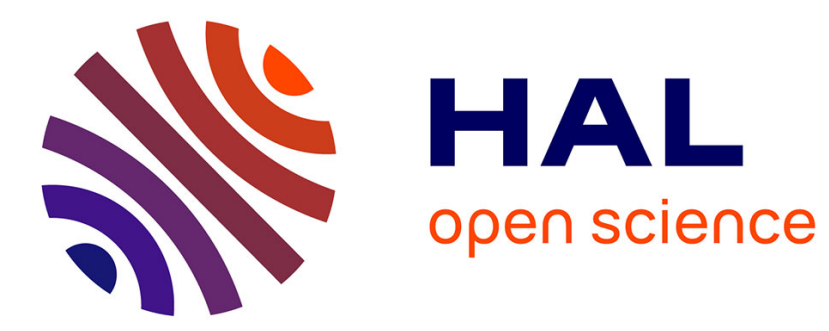

\title{
Global linear stability of a model subsonic jet
}

Xavier Garnaud, Lutz Lesshafft, Patrick Huerre

\section{To cite this version:}

Xavier Garnaud, Lutz Lesshafft, Patrick Huerre. Global linear stability of a model subsonic jet. AIAA Paper, 2011, pp.2011-3608. hal-00716674

\section{HAL Id: hal-00716674 \\ https://hal.science/hal-00716674}

Submitted on 11 Jul 2012

HAL is a multi-disciplinary open access archive for the deposit and dissemination of scientific research documents, whether they are published or not. The documents may come from teaching and research institutions in France or abroad, or from public or private research centers.
L'archive ouverte pluridisciplinaire $\mathbf{H A L}$, est destinée au dépôt et à la diffusion de documents scientifiques de niveau recherche, publiés ou non, émanant des établissements d'enseignement et de recherche français ou étrangers, des laboratoires publics ou privés. 


\title{
Global linear stability of a model subsonic jet
}

\author{
X. Garnaud* and L. Lesshafft ${ }^{\dagger}$ and P. Huerre ${ }^{\ddagger}$ \\ Ecole Polytechnique - CNRS, 91128 Palaiseau, France
}

\begin{abstract}
The global stability of a subsonic jet is investigated using a model base flow designed to fit experimental results for turbulent mean flows. Eigenmodes are computed for axisymmetric perturbations in order to investigate the nature of typically observed large-scale coherent oscillations ("preferred mode"). We do not find evidence that this preferred mode corresponds to the least damped global mode. Non-modal stability is also considered through the computation of optimal perturbations. Although non-axisymmetric perturbations (in particular for azimuthal wavenumber $m=1$ ) are subject to larger transient growth, these reach their peak amplitude far downstream of the potential core, and therefore they are less likely to be observed.
\end{abstract}

\section{Nomenclature}

$\begin{array}{ll}r, x, \theta & \text { radial, axial and azimuthal cylindrical coordinates } \\ t & \text { time } \\ m & \text { azimuthal wave number } \\ \boldsymbol{u} & \text { Velocity vector } \\ \rho, T, p, e, E, s & \text { density, temperature, pressure, internal energy, total energy and entropy } \\ c & \text { speed of sound } \\ \mathrm{Re}, \mathrm{Ma}, \mathrm{Pr}, \mathrm{St}, S & \text { Reynolds, Mach, Prandtl and Strouhal numbers, axis to infinity density ratio } \\ \mu, C_{p}, \gamma, \kappa & \text { viscosity, specific heat at constant pressure, adiabatic index and thermal conductivity } \\ R & \text { jet radius } \\ \Theta & \text { momentum thickness }\end{array}$

Subscript

$r, x, \theta$

$(r),(i)$

radial, axial and azimuthal vector components

$0, \infty$

real and imaginary parts of complex numbers

value on the axis and in the fluid at rest

Superscript

$\begin{array}{ll}* & \text { dimensional value } \\ b & \text { base flow fields }\end{array}$

\section{Introduction}

Aero-acoustic noise generation by subsonic jets may be attributed to two classes of source mechanisms. First, fine-scale turbulence radiates a broad band noise, radiating similarly in all directions with respect to the jet axis. Second, large-scale coherent structures are observed in turbulent jets over several jet diameters downstream of the nozzle, as first described by Crow \& Champagne. ${ }^{13}$ These structures reach a peak amplitude at a Strouhal number of the order of $0.2-0.5$, based on the jet diameter. These coherent structures radiate most of the acoustic energy ${ }^{9,16}$ and constitute the subject of this study. The prefered mode

*PhD student, LadHyX, Ecole Polytechnique, 91128 Palaiseau Cedex, France.

${ }^{\dagger}$ CNRS Research Scientist, LadHyX, Ecole Polytechnique, 91128 Palaiseau Cedex, France.

${ }^{\ddagger}$ CNRS Director of Research and Professor, LadHyX, Ecole Polytechnique, 91128 Palaiseau Cedex, France.

Copyright (c) 2011 by LadHyX, Ecole Polytechnique - CNRS. Published by the American Institute of Aeronautics and Astronautics, Inc. with permission. 
is described as axisymmetric and interpreted as a slightly damped eigenmode. ${ }^{13}$ In order to understand this phenomenon, linear stability studies have been performed, under the assumption of a paralle ${ }^{5}$ and a slowly diverging ${ }^{11,29}$ base flow. These early results are in reasonably good agreement with experimental data, and discrepancies are attributed to non-parallel effects in particular due to the presence of the jet nozzle. More recently, Gudmundsson \& Colonius ${ }^{18}$ treated this problem using Parabolized Stability Equations (PSE). Within this approximation, it is assumed that information only propagates downstream and that the effect of non-parallelism is weak. This method therefore does not directly yield the acoustic field, although it can be recovered from an acoustic analogy. ${ }^{10}$ Owing to the increasing computing power modal and non-modal studies of fully non-parallel flows have recently become accessible, in particular through the use of matrixfree methods..$^{7,15,17,22,27}$ In the present study, a modal stability analysis of axisymmetric perturbations is performed in order to elucidate the physical origin of the preferred mode. Isothermal jet flows have been found to be convectively unstable, ${ }^{26}$ and such flows act as noise amplifiers. They are globally stable in the sense that all perturbations eventually decay in a linear framework. Perturbation can however be strongly amplified at finite time, which may lead to amplitude saturation in a non-linear setting. Non-modal growth is studied here in the framework of optimal perturbations, i.e. the perturbations that give rise to the largest energy growth over a finite time $T$.

In "global" instability studies, a steady laminar solution of the Navier-Stokes equations ${ }^{23,24}$ is often used as the base flow (a boundary layer approximation is sometimes used as a cheaper alternative ${ }^{2,28}$ ), so that the linear stability study rigorously describes the dynamics of infinitesimal perturbations in a laminar flow. This approach is however not expected to give realistic results when applied to jets, as turbulence plays a major role in the flow development; laminar steady states are in particular unable to reflect the typical size of the potential core, of the order of four to five jet diameters. Turbulence modeling should be added in order to fully capture the base flow development. Crouch et al. ${ }^{12}$ considered the stability of a flow not described by the Navier-Stokes equations but by a RANS model. In the present paper, a composite approach is pursued. A model base flow inspired by Monkewitz \& $\mathrm{Sohn}^{26}$ is considered, on top of which large scale perturbations are considered. Gudmundsson \& Colonius ${ }^{18}$ used a similar approach: based on a fit of PIV measurements, they derived an analytical model for a time-averaged flow. A PSE stability study of this mean flow resulted in good agreement with experiments.

\section{Flow configuration and dimensionless variables}

We consider a jet issuing from of a semi-infinite cylindrical duct of radius $R^{*}$, with a velocity $U_{0}^{*}$ on the jet axis. The jet exits into fluid at rest, characterized by its density $\rho_{\infty}^{*}$ and its temperature $T_{\infty}^{*}$. The fluid is assumed to be a perfect Newtonian gas; furthermore, its adiabatic index $\gamma=1.4$, thermal conductivity $\kappa^{*}$, specific heat at constant pressure $C_{p}^{*}$ and viscosity $\mu^{*}$ are assumed to be constant.

Non-dimensional variables, denoted without asterisks, are defined with respect to $R^{*}, U_{0}^{*}, \rho_{\infty}^{*}$ and $T_{\infty}^{*}$ as reference length, velocity, density and temperature scales. With this normalization, natural choices for the Reynolds, Mach and Prandtl numbers are

$$
\operatorname{Re}=\frac{U_{0}^{*} R^{*} \rho_{\infty}^{*}}{\mu^{*}}, \quad \quad \mathrm{Ma}=\frac{U_{0}^{*}}{c_{\infty}^{*}}, \quad \operatorname{Pr}=\frac{\mu^{*} C_{P}^{*}}{\kappa^{*}},
$$

where $c_{\infty}^{*}=\sqrt{\gamma r^{*} T_{\infty}^{*}}$ denotes the ambient speed of sound. In terms of dimensionless variables, the equation of state becomes

and the internal energy $e$ is given by

$$
p=\frac{\rho T}{\gamma \mathrm{Ma}^{2}}
$$

$$
e=\frac{T}{\gamma(\gamma-1) \mathrm{Ma}^{2}}
$$

Entropy may be defined as

$$
s=\frac{1}{\gamma(\gamma-1) \mathrm{Ma}^{2}}[\log (p)-\gamma \log (\rho)]
$$

Frequencies $f^{*}$ can be non-dimensionalized either as a Strouhal number (based on the jet diameter) or as a dimensionless circular frequency $\omega$, according to

$$
\mathrm{St}=\frac{2 f^{*} R^{*}}{U_{0}^{*}}=\frac{\omega}{\pi} .
$$


The flow is described in cylindrical coordinates $r, x, \theta, x=0$ corresponding to the nozzle exit. Using the above non-dimensionalization, the compressible Navier-Stokes equations are given by Sandberg. ${ }^{32}$ They are linearized in order to perform the stability studies described below.

\section{Model jet flow}

Neither boundary layer approximation nor steady-state solutions of the Navier-Stokes equations can reflect the rapid streamwise development observed in turbulent jets. For the purpose of this study, a parametric model of a turbulent jet is adapted from $\mathrm{Sohn}^{35}$ (see also Monkewitz \& $\mathrm{Sohn}^{26}$ ) and schematically represented in figure 1 . The model is designed to match experimentally measured mean flows in the subsonic regime. As the original manuscript is not widely available, the free-jet model is reviewed in $\S$ III.A. Downstream of the nozzle, experimental studies indicate that the potential core typically extends over 8 jet radii, and that in this region the shear layer initially spreads linearly with $x$ while the velocity on the axis remains approximately constant. Further downstream, velocity profiles approximated as being Gaussian, and they continue to spread radially.. The flow inside the inlet duct is considered to be parallel (§ III.B). A smooth transition between the pipe flow and the free jet is realized in a small matching region downstream of the nozzle (§ III.C).

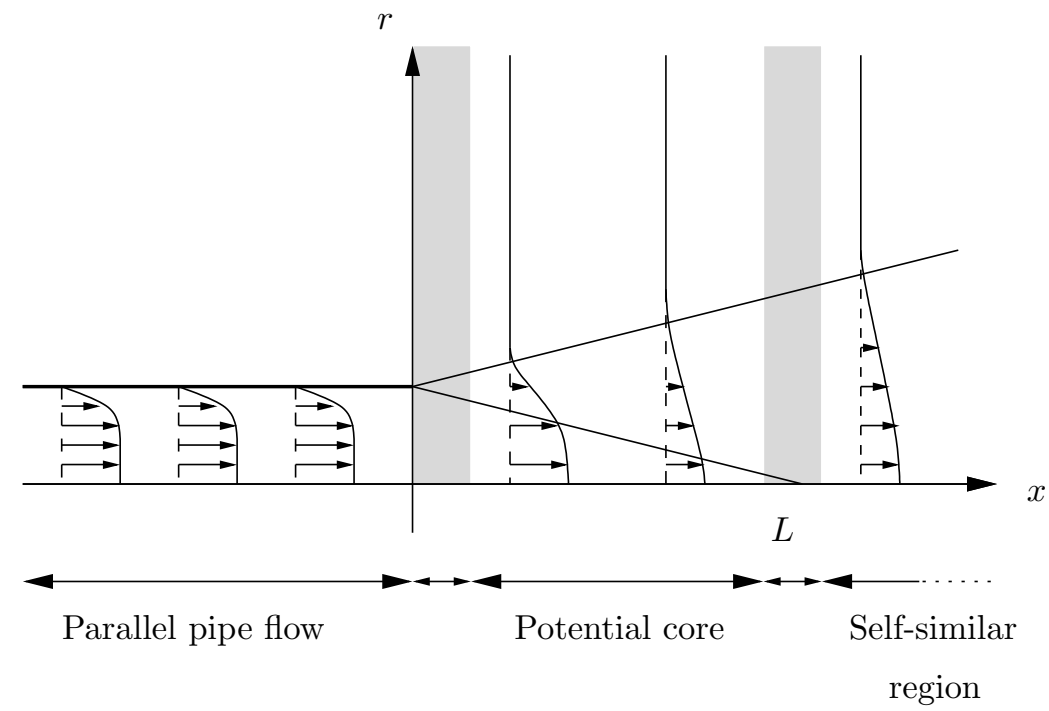

Figure 1. Model base flow. The parallel pipe flow region, the potential core and the self similar region are connected by smooth transitions, indicated by shaded regions in the figure.

\section{III.A. Free jet}

The parametric model of a turbulent mean jet flow developed by Sohn ${ }^{35}$ is used in this study. A family of profiles is described, characterized by the density ratio at the inlet

$$
S_{0}=\frac{\rho(0,0)}{\rho_{\infty}}
$$

and the Mach number. A third parameter $\epsilon_{0}$, characterizing the shear layer thickness at the outlet is introduced in the present study.

Explicit analytical formulas are given for the length of the potential core and the evolution of the jet axial velocity $u_{x}$ on the centerline. The shape of the axial velocity profile is also given up to a radial scale $\delta(x)$ that remains to be determined. At each axial position, the relationship between density and axial velocity profiles is also given as a function of the density on the jet axis $S(x) . \delta$ and $S$ are chosen such that momentum and energy flux are conserved throughout the jet.

Potential core The length of the potential core is prescribed as

$$
L=8+2.5 \log \left(S_{0}\right)
$$


(a)

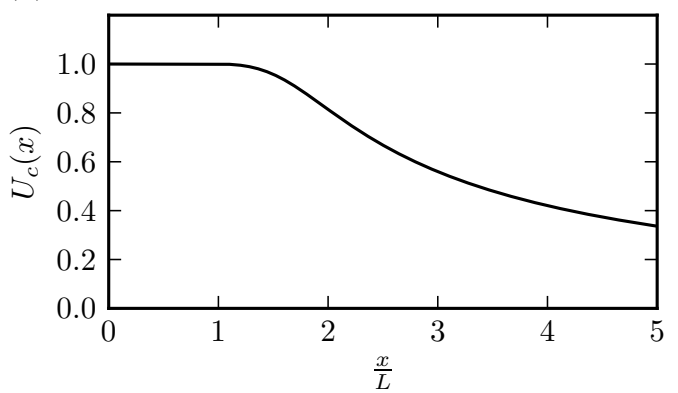

(b)

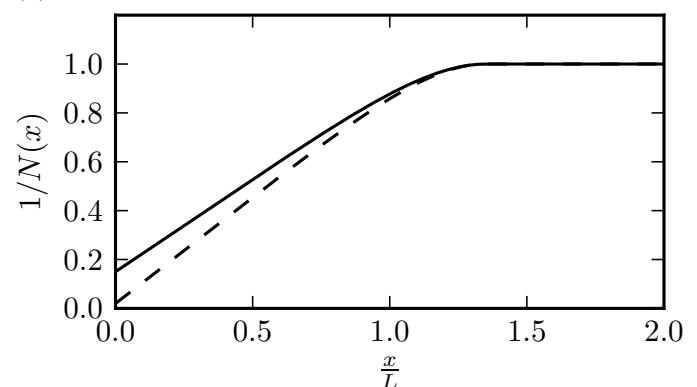

Figure 2. (a): Distribution of axial velocity $U_{c}(x)$ given by (3). (b) Distribution of the shear layer thickness parameter $N(x)$ given by (4) (dashed line) and by (7) with $\epsilon_{0}=0.15$ (solid line).

Axial VELocity PRofile Axial velocity profiles are assumed to be of the form

$$
u_{x}(r, x)=U_{c}(x) F\left(\frac{r}{\delta(x)}, x\right)
$$

with

$$
F(\eta, x)=\left\{1+\left[\exp \left(\eta^{2} \log (2)\right)-1\right]^{N(x)}\right\}^{-1} .
$$

The quantity $U_{c}(x)$ denotes the velocity on the jet center-line: in order to fit experimental measurements it is assumed to be given by

$$
U_{c}(x)= \begin{cases}1 . & \frac{x}{L} \leq 1 . \\ 1.682\left[\left(\frac{x}{L}\right)^{7}+37.1\left(\frac{x}{L}\right)^{-0.189}\right]^{-1 / 7} & \frac{x}{L}>1 .\end{cases}
$$

The parameter $N(x)$ governs the smoothness of the profile: $N \rightarrow \infty$ corresponds to a cylindrical vortex sheet, while $N=1$ corresponds to a gaussian velocity distribution. The streamwise variation of $N$ is given by

$$
N(x)=\left\{\begin{array}{cc}
{\left[0.02+0.869\left(\frac{x}{L}\right)-0.031\left(\frac{x}{L}\right)^{6.072}\right]^{-1}} & \frac{x}{L} \leq 1.35 . \\
1 . & \frac{x}{L}>1.35
\end{array}\right.
$$

and $U_{c}$ and $N$ are represented as functions of $x$ in figure 2 .

Pressure FIELD Pressure is assumed to be constant,

$$
p=\frac{1}{\gamma \mathrm{Ma}^{2}} .
$$

DENSITY / TEMPERATURE Distribution The relation between temperature and velocity is taken from Schlichting: ${ }^{33}$

$$
\frac{1 / \rho(r, x)-1}{1 / S(x)-1}=\left(\frac{u_{x}(r, x)}{U_{c}(x)}\right)^{\sigma(x)}=F\left(\frac{r}{\delta(x)}, x\right)^{\sigma(x)},
$$

where the distribution of $\sigma$ is given by

$$
\sigma(x)= \begin{cases}1 . & \frac{x}{L} \leq 0.64 \\ 0.8+0.2 \cos \left(2.49 \frac{x}{L}-1.60\right) & 0.64<\frac{x}{L} \leq 1.90 \\ 0.6 & \frac{x}{L}>1.90\end{cases}
$$

to match experiments. 
Momentum And EnERgy COnSERvation The streamwise development of $\delta$ and $S$ is not prescribed: these are computed so that momentum and energy fluxes through sections perpendicular to the jet axis are constant:

$$
\int_{0}^{\infty} \rho u_{x}^{2} r \mathrm{~d} r=\mathcal{M}
$$

and

$$
\int_{0}^{\infty} \rho u_{x}\left[\frac{T-1}{(\gamma-1) \mathrm{Ma}^{2}}+\frac{1}{2} u_{x}^{2}\right] r \mathrm{~d} r=\mathcal{E}
$$

These expressions can be rewritten as

$$
\mathcal{M}=\delta(x)^{2} U_{c}(x)^{2} \int_{0}^{\infty} \frac{F(\eta, x)^{2}}{1+(1 / S(x)-1) F(\eta, x)^{\sigma(x)}} \eta \mathrm{d} \eta
$$

and

$$
\mathcal{E}=\delta(x)^{2} \int_{0}^{\infty} \frac{1}{2} \frac{U_{c}(x)^{3} F(\eta, x)^{3}}{1+(1 / S(x)-1) F(\eta, x)^{\sigma(x)}}+\frac{(1 / S(x)-1)}{(\gamma-1) \mathrm{Ma}^{2}} \frac{U_{c}(x) F(\eta, x)^{1+\sigma(x)}}{1+(1 / S(x)-1) F(\eta, x)^{\sigma(x)}} \eta \mathrm{d} \eta
$$

The invariants $\mathcal{M}$ and $\mathcal{E}$ are determined by their values at $x=0$, where $\delta(0)=1$ and $S(0)=S_{0}$. The ratio $\mathcal{M} / \mathcal{E}$ is a function of $S(x)$ alone and can be solved numerically. This value is then used to obtain $\delta(x)$ from momentum and energy conservation.

THICKNESS OF THE SHEAR LAYER The thickness of the shear layer can be estimated through the momentum thickness

$$
\Theta(x)=\int_{0}^{\infty} \frac{\rho(r, x) u_{x}(r, x)}{\rho(0, x) u_{x}(0, x)}\left(1-\frac{u_{x}(r, x)}{u_{x}(0, x)}\right) r \mathrm{~d} r .
$$

which is related to the distribution of $N(x)$. The variations of $N$ in the potential core are such that:

- the shear layer thickness is of the order of 0.02 at the nozzle,

- the shear-layer spreading is approximately linear in the potential core,

- it matches a Gaussian distribution at $x=1.35 L: N$ and its first derivative are continuous.

From a numerical point of view, the treatment of such a shear layer would be expensive. The only modification to the free jet model of Sohn reviewed above is to prescribe $N$ of the form

$$
N(x)=\left[\epsilon_{0}+a x+b x^{6}\right]^{-1}, \quad x \leq 1.35 L .
$$

The parameter $\epsilon_{0}$ is chosen as $\epsilon_{0}=0.15$, which gives $\Theta=0.054$ at $x=0$ (this value is modified by the presence of the pipe flow as explained in in $\S$ III.B). The coefficients $a$ and $b$ are determined in order to ensure continuity of $N$ and $N^{\prime}$ at $x=1.35 L$. The new distribution of $N$ is represented in figure 2 together with the one given by (4); they have similar behavior except for the value at $x=0$.

\section{III.B. Pipe flow}

PARAllel PIPE Flow The axial velocity profile in the pipe is assumed to be of the form

$$
u_{x}(r, x)=\left\{\begin{array}{cc}
2\left(1+\left[\exp \left(r^{2} \log (2)\right)-1\right]^{N_{1}}\right)^{-1}-1 & r \leq 1 . \\
0 & r>1 .
\end{array}\right.
$$

The parameter $N_{1}$ characterizes the maximum slope of the velocity profile. It should be of the order of $\epsilon_{0}^{-1}$ to continuously match (8) with the jet flow velocity distribution given by (2). An adiabatic pipe is considered, therefore uniform temperature, density and pressure distribution are assumed inside and outside the pipe. The density is respectively equal to $S_{0}$ for $r \leq 1$ and 1 for $r>1$. This pipe flow profile defines the momentum and energy fluxes $\mathcal{M}$ and $\mathcal{E}$ to be conserved throughout the jet. The resulting distributions of $\delta$ and $S$ are represented in figure 3 . 
(a)

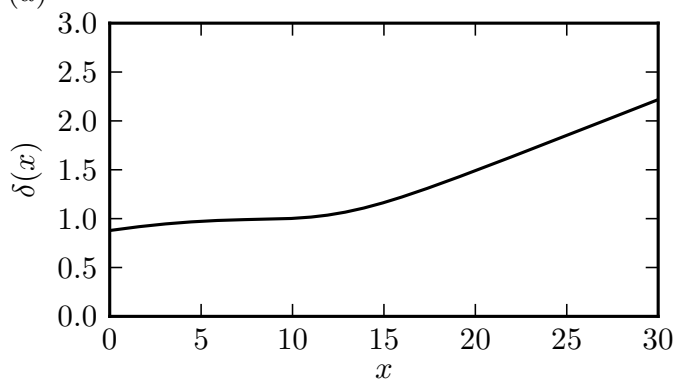

(b)

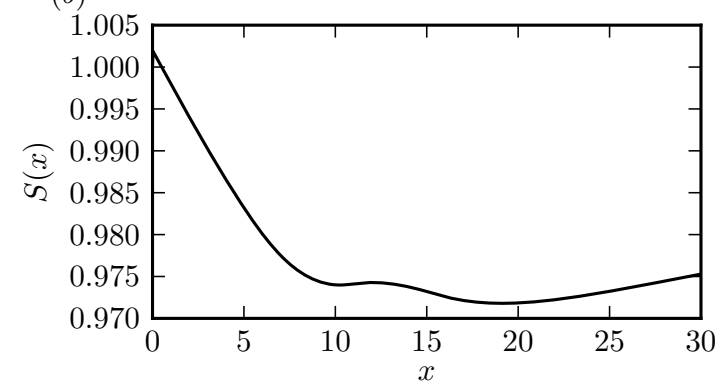

Figure 3. Application of momentum and energy conservation for $\epsilon_{0}=0.15, \mathbf{M a}=0.75$ and $S_{0}=1$. A value of $N_{1}=0.6 / \epsilon_{0}$ was chosen to ensure smoothness of the momentum thickness. (a): jet radial scale. (b): local density ratio.

\section{III.C. Matching}

MATChing OF PIPE AND JET FLOW Let $\phi$ denote any of the flow fields, $\rho, u_{x}$ or $T$. If $\phi^{j}(r, x)$ is the field in the jet (given in $\S$ III.A) and $\phi^{p}(r)$ the same field in the pipe (given in $\S I I I . B$ ), a continuous matching between both is achieved as follows. Given a transition length $x_{t}, \phi_{b}$ is defined in the entire domain as

$$
\phi_{b}(r, x)=\left\{\begin{array}{cl}
\phi^{p}(r) & x \leq 0, \\
{\left[\left(1-\psi\left(\frac{x}{x_{t}}\right)\right] \phi^{p}(r)+\psi\left(\frac{x}{x_{t}}\right) \phi^{j}(r, x)\right.} & 0<x \leq x_{t}, \\
\phi^{j}(r, x) & x_{t}<x,
\end{array}\right.
$$

where $\psi$ is a smooth function so that $\psi(0)=0$ and $\psi(1)=1$. The values of $x_{t}$ and $N_{1}$ are chosen such that the evolution of the momentum thickness is continuous. The resulting axial velocity field is represented in figure 4 .

(a)

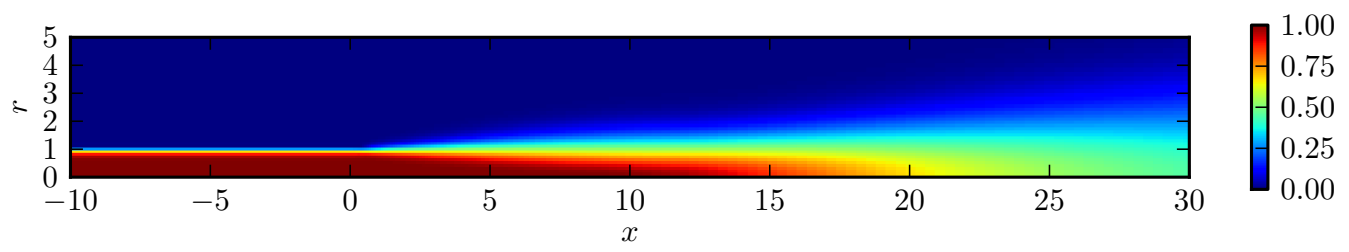

(b)

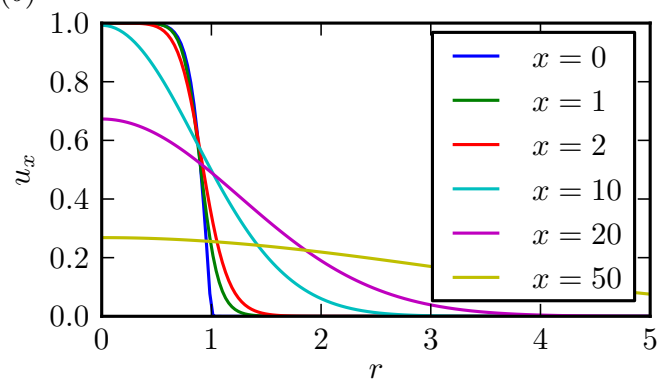

(c)

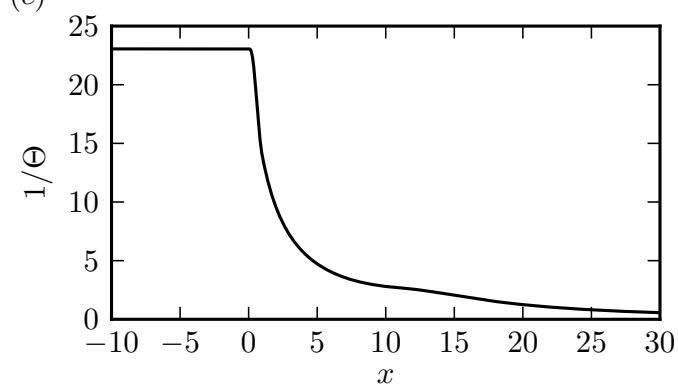

Figure 4. Base flow for $\epsilon_{0}=0.15, \mathbf{M a}=0.75$ and $S_{0}=1$ and $x_{t}=1$. . A value of $N_{1}=0.6 / \epsilon_{0}$ was chosen to ensure smoothness of the momentum thickness. $(a)$ : isocontours of axial velocity. (b): axial velocity profiles at various $x$ locations. $(c)$ : momentum thickness.

RADIAL VELOCITY: MASS CONSERVATION Only momentum and energy fluxes across sections perpendicular to the jet axis are conserved. Mass conservation is imposed locally, in order to obtain a radial velocity field $u_{0, r}$ from the previously computed axial velocity and density fields using local mass conservation:

$$
\frac{\partial \rho_{b} u_{0, x}}{\partial x}+\frac{\partial \rho_{b} u_{0, r}}{\partial r}+\frac{\rho_{b} u_{b, r}}{r}=0
$$




\section{Linear stability analysis}

\section{IV.A. Assumptions}

The model flow defined in section III is used as a base flow for a linear stability analysis, although it is not a steady solution of the Navier-Stokes equations. Let $L$ be the Navier-Stokes operator linearized about this base state. The dynamics of small perturbations, characterized by the state vector $\boldsymbol{q}=(\rho, \boldsymbol{u}, E)^{T}$, are assumed to be governed by

$$
\frac{\mathrm{d} \boldsymbol{q}}{\mathrm{d} t}=L \boldsymbol{q}
$$

Given the axisymmetry of the base flow, perturbations are decomposed into azimuthal Fourier modes

$$
\boldsymbol{q}(r, x, \theta ; t)=\sum_{m} \boldsymbol{q}_{m}(r, x ; t) \exp (\mathrm{i} m \theta)
$$

which can be treated independently. Let their dynamics be governed by

$$
\frac{\mathrm{d} \boldsymbol{q}_{m}}{\mathrm{~d} t}=L_{m} \boldsymbol{q}_{m}
$$

\section{IV.B. Discretization}

Equation (11) is discretized on an orthogonal grid. For the present study, the domain $-110 \leq x \leq 170$, $0 \leq r \leq 170$ was discretized using $384 \times 768$ points. Grid stretching is kept below $4 \%$ such that numerical dispersion remains limited. Explicit centered $5^{t h}$-order finite differences in combination with a spatial filtering scheme are used for the spatial derivatives (see Berland $e t a l .{ }^{6}$ ). The role of the spatial filter is to suppress the numerical instabilities arising from the use of a centered finite-difference scheme. The choice of discretization scheme is dictated by the need to accurately represent acoustic wave propagation. Non-reflecting boundary conditions given by Bogey \& Bailly ${ }^{8}$ are used together with sponge regions, as indicated in figure 5 . Time stepping is performed using a $3^{\text {rd }}$-order Runge-Kutta algorithm.

For the optimization procedures described later, the adjoint operator is needed. A discrete adjoint, i.e. the Hermitian transpose of the direct discretized operator, is used. It is applied in a matrix-free framework, as described by Fosas de Pando et al. ${ }^{14}$

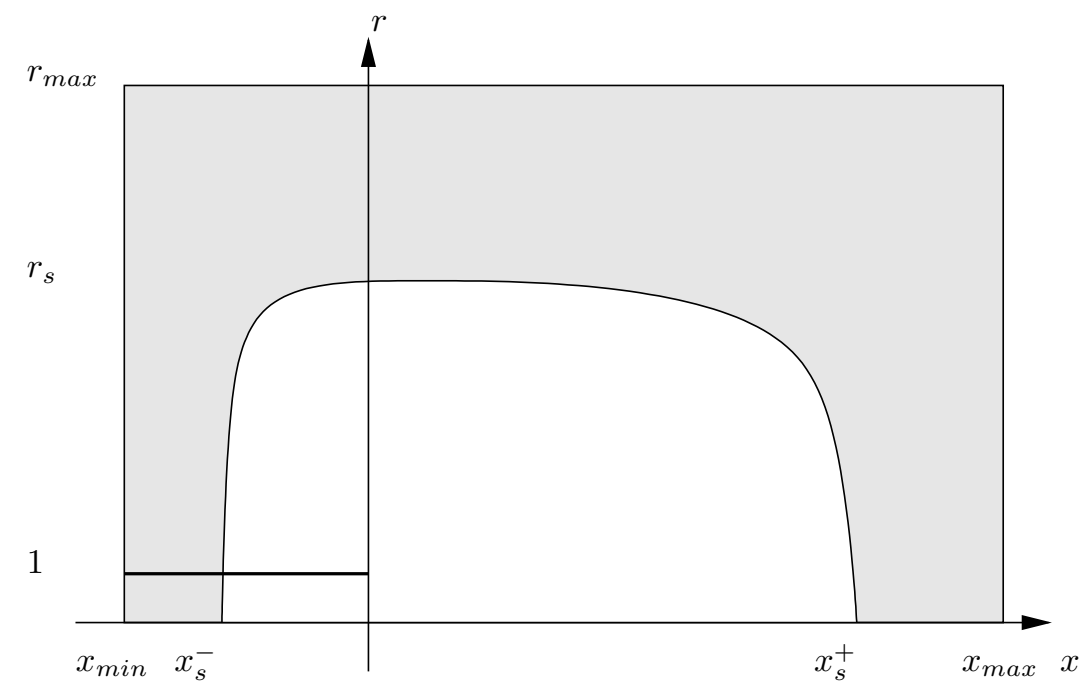

Figure 5. Computational domain. Sponge layers are represented in gray. Results are presented here for $x_{m i n}=-110$, $x_{\max }=170$ and $r_{\max }=170$.

\section{IV.C. Global modes}

Method Global modes $\boldsymbol{q}$ are temporal eigenmodes of the linearized Navier-Stokes equations. They verify

$$
L_{m} \boldsymbol{q}=\mathrm{i} \omega \boldsymbol{q}
$$


where $\omega=\omega_{(r)}+\mathrm{i} \omega_{(i)}$ is the complex eigenfrequency. The classical local stability approach is based on the assumption of a parallel base flow, so that perturbations can be decomposed into independent Fourier modes in the azimuthal and radial directions. Only the radial direction is discretized. This results in eigenvalue problems of moderate sizes that can be solved directly. In the global framework, the base flow is no longer assumed to be parallel and boundary conditions are taken into account in both the streamwise and crossstream directions. This results in a two-dimensional eigenvalue problem. The dimension of such a problem is easily on the order of $10^{6}$ degrees of freedom, making the use of direct methods impossible. A limited number of eigenvectors can however be computed using variants of the Arnoldi algorithm. ${ }^{20,21}$ This method extracts the dominant eigenvalues, i.e. those with the largest magnitude. If it were to be applied directly to the linearized Navier-Stokes operator, it would extract modes with either a large frequency or a large decay rate, all of these being numerical artifacts. Spectral transformations are therefore employed to transform the operator $L_{m}$ into another operator $\mathcal{T}\left(L_{m}\right)$ so that its largest amplitude modes are physically relevant.

Edwards et al. ${ }^{15}$ introduced the time stepper technique in the field of flow analysis. By considering the propagator $\mathcal{T}\left(L_{m}\right)=\exp \left(\Delta T L_{m}\right)$, this method allows to compute the least stable modes of $L_{m}$ by simply coupling a time stepper to an eigenvalue solver. In the present case of a globally stable flow (i.e. with only stable modes) in an unbounded domain, this method would only capture modes belonging to continuous acoustic and vortical branches with near-zero decay rates (see Schmid \& Henningson ${ }^{34}$ for details) .

In order to focus on more specific regions of the spectrum, variants of the "shift-invert" spectral transformation ${ }^{21}$ have been applied to flow stability analysis. ${ }^{2,24,28}$ In these methods, the spectral transformation is of the form $\mathcal{T}\left(L_{m}\right)=\left(L_{m}-\sigma \mathrm{Id}\right)^{-1}$. Systems of the form $\left(L_{m}-\sigma \mathrm{Id}\right) \boldsymbol{x}=\boldsymbol{b}$ need to be solved repeatedly. This has been done by direct methods in dense ${ }^{2}$ and sparse ${ }^{3,4,24}$ frameworks, as well as by iterative methods. ${ }^{22,31}$ All these methods are computationally expensive (LU decomposition for direct solvers, preconditioners for iterative solvers ), in particular in terms of memory requirements. Furthermore, they require the explicit assembly of the matrix of the operator $L_{m}$ (or an approximation for preconditioning).

Inspired by both these methods and Selective Frequency Damping, ${ }^{1}$ originally introduced for unstable base flow computation, a new spectral transformation has been introduced. ${ }^{17}$ First, the operator $L_{m}$ is relaxed in order to stabilize modes away from a shift frequency $\omega_{0}$. The resulting composite operator is

$$
\mathcal{F}\left(L_{m}\right) \equiv\left(\begin{array}{c|c}
L_{m}-\tau \mathrm{Id} & \tau \mathrm{Id} \\
\hline \tau \mathrm{Id} & \left(-\mathrm{i} \omega_{0}-\tau\right) \mathrm{Id}
\end{array}\right)
$$

If the dimension of $L_{m}$ is $N \times N$, the dimension of $\mathcal{F}$ is therefore $2 N \times 2 N$. It can be shown that if $\boldsymbol{Q}$ is a mode of $\mathcal{F}\left(L_{m}\right)$, then its first $N$ components form a mode $\boldsymbol{q}$ of $L_{m}$. Furthermore, the relaxation procedure plays the expected role and modes with frequency outside a band of width $\tau$ centered around $\omega_{0}$ are stabilized. Coupling the propagator of $\mathcal{F}, \mathcal{T}\left(L_{m}\right)=\exp \left[\Delta T \mathcal{F}\left(L_{m}\right)\right]$, to an eigenvalue solver therefore provides a way to compute selected eigenmodes in a matrix-free and scalable way, and with limited memory requirements.

RESUlts Eigenmodes have been computed for $0.5 \leq \omega_{(r)} \leq 1.5(0.15 \leq \mathrm{St} \leq 0.5)$ for axisymmetric perturbations. Only $m=0$ is considered here as the preferred mode has been described as being axisymmetric. The resulting eigenvalue spectrum is displayed in figure 6 . The decay rate of the modes $-\omega_{(i)}$ increases with the real frequency $\omega_{(r)}$. For three particular modes, labeled 1 to 3 in figure 6 , the azimuthal vorticity field is displayed in figure 7 . The amplitude of the perturbations grows in the streamwise direction until they reach a maximum, after which they decay slowly. For the lowest frequencies computed, this maximum is reached inside the sponge layer, the limit of which is indicated by the dashed line. As the frequency decreases, the position of maximum intensity moves upstream and the carrier wavelength decreases. This is consistent with a phase velocity of the order of half the centerline velocity. For the modes of highest frequencies presented in figure 7, vortical structures in the self-similar region are still correctly resolved throughout the entire domain. It is also interesting to notice that modes start to exhibit significant vorticity fields (about one tenth of the maximum intensity) in the shear layer immediately downstream of the nozzle.

Figure 8 displays the dilatation field $(\boldsymbol{\nabla} \cdot \boldsymbol{u})$ associated with mode number 3 in figure 6 . This acoustic field is very strongly dependent on the boundary conditions, and it is likely to still be affected by spurious reflections. Indeed, the temporal decay of the modes translates into an exponential spatial growth of the acoustic amplitude away from the source region, which imposes severe accuracy requirements for the nonreflecting boundary conditions. In the absence of such outward exponential growth, as for instance in the optimal perturbation computations presented in the next section, the implemented boundary conditions let acoustic waves exit the computational domain with minimal reflection. Tests have shown that neither the 


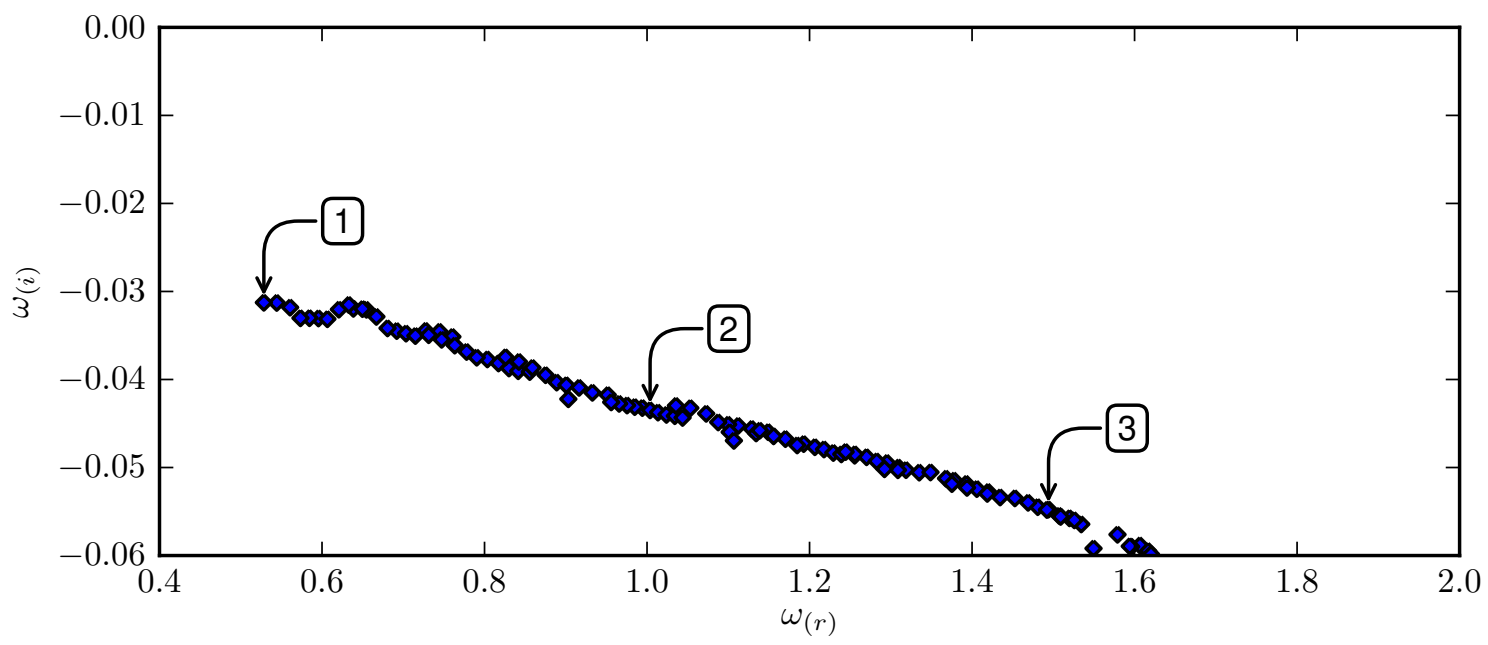

Figure 6. Eigenvalue spectrum for axisymmetric perturbations.
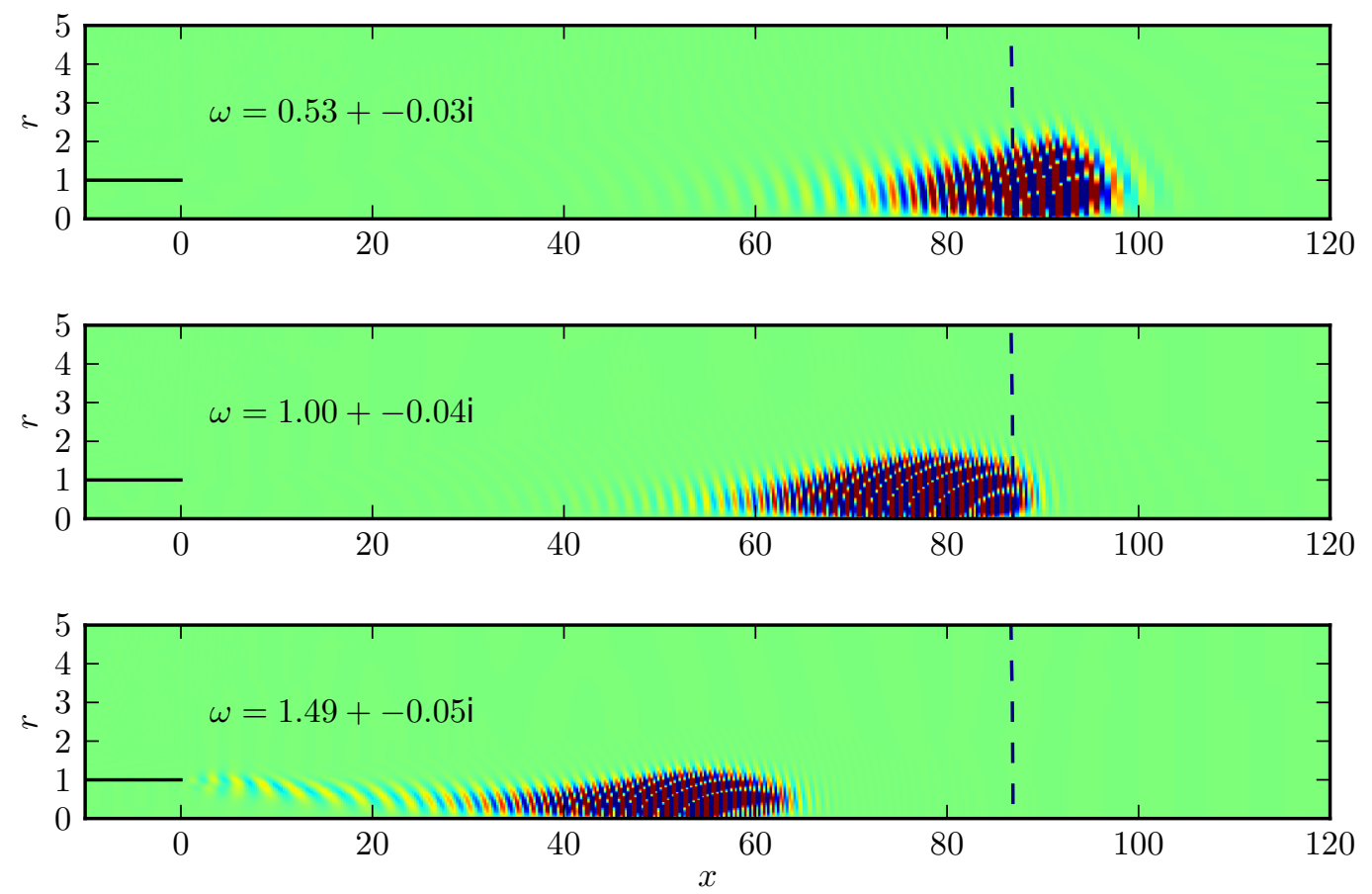

Figure 7. Azimuthal vorticity fields of global modes indicated by labels in figure 6 . The limit of the sponge layer in indicated by the dashed line.

spectrum nor the vortical near-field structures of the global modes are significantly affected by the presence of these spurious reflections. 


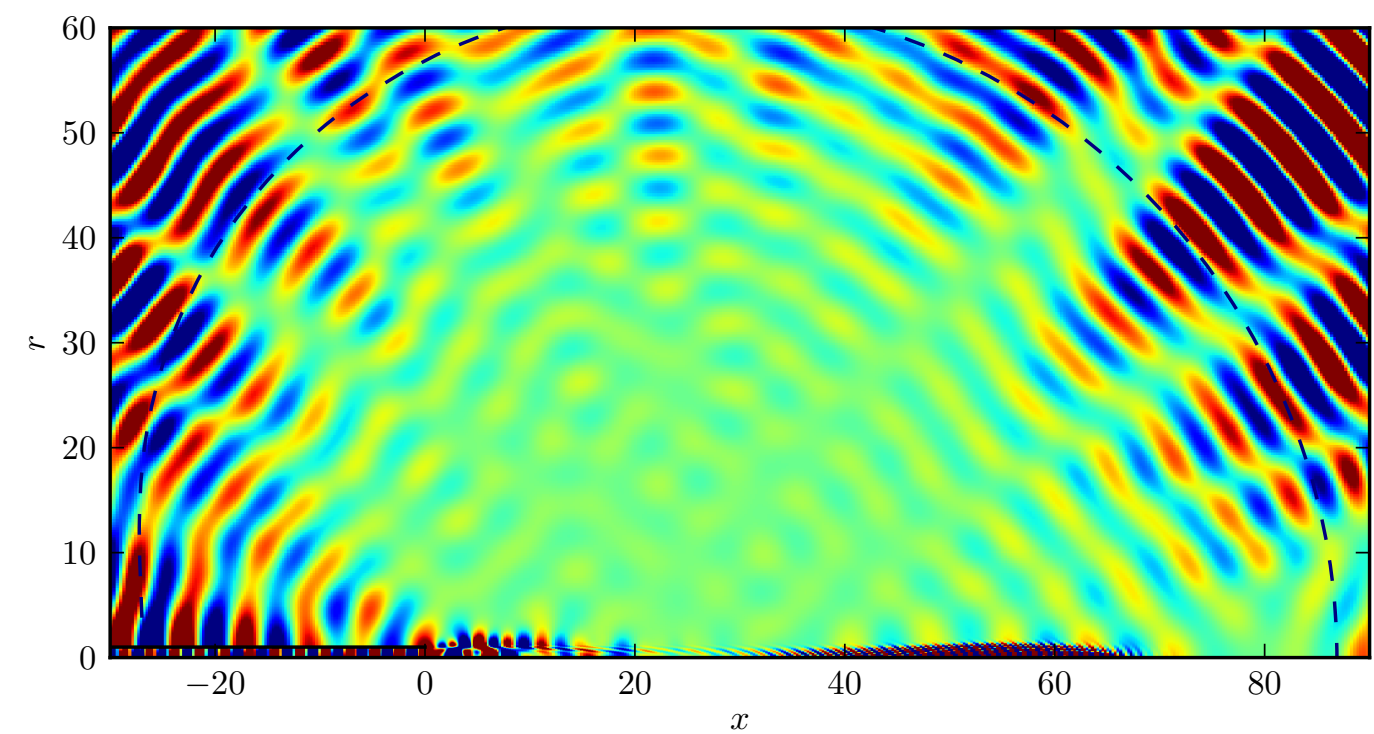

Figure 8. Dilatation field $(\nabla \cdot u)$ for mode number 3 in figure 6 . The limit of the sponge layer in indicated by the dashed line. Only part of the computational domain is shown.

\section{IV.D. Optimal perturbations}

In order to measure disturbances from the base flow profile, Hanifi et al. ${ }^{19}$ introduced the norm

$$
\left\|\boldsymbol{q}_{m}\right\|^{2}=\iint_{\Omega}\left(\rho_{b}\left|u_{m}\right|^{2}+\frac{p_{b}}{\rho_{b}}\left|\rho_{m}\right|^{2}+\frac{\rho_{b}^{3}}{\gamma^{2}(\gamma-1) \mathrm{Ma}^{4} p_{b}}\left|T_{m}\right|^{2}\right) r \mathrm{~d} r \mathrm{~d} x
$$

designed such that its contribution from compression work is zero. Equivalently, the norm can be expressed as

$$
\left\|\boldsymbol{q}_{m}\right\|^{2}=\iint_{\Omega}\left(\rho_{b}\left|u_{m}\right|^{2}+\mathrm{Ma}^{2}\left|p_{m}\right|^{2}+p_{b} \rho_{b} \gamma(\gamma-1) \mathrm{Ma}^{4}\left|S_{m}\right|^{2}\right) r \mathrm{~d} r \mathrm{~d} x
$$

Using the above norm, optimal perturbations are computed (see Reddy \& Henningson ${ }^{30}$ for details). For an objective time $T$, these represent the initial condition of (10) which yields the maximum energy gain at time $T$ :

$$
\begin{aligned}
\boldsymbol{q}_{\text {max }}^{T, m} & =\arg \max \frac{\left\|\exp \left(T L_{m}\right) \boldsymbol{q}_{m}\right\|_{N_{1}}}{\left\|\boldsymbol{q}_{m}\right\|_{N_{2}}}, \\
G_{m}(T) & =\max \frac{\left\|\exp \left(T L_{m}\right) \boldsymbol{q}_{m}\right\|_{N_{1}}}{\left\|\boldsymbol{q}_{m}\right\|_{N_{2}}} .
\end{aligned}
$$

In the above equations, $N_{2}$ should be a norm so that the objective function possesses a finite maximum. The norm $N_{1}$ by contrast does not need to be definite. In the present case, $N_{2}$ is the discretization of the norm introduced in (14) with $\Omega$ being the entire numerical domain. For $N_{1}, \Omega$ only encompasses the physical domain, i.e. sponge layers do not contribute to the norm.

Numerical implementation Let $M_{1}$ and $M_{2}$ be matrices such that

$$
\left\|\boldsymbol{q}_{m}\right\|_{N_{1}}=\boldsymbol{q}_{m}^{\dagger} M_{1}^{\dagger} M_{1} \boldsymbol{q}_{m} \quad\left\|\boldsymbol{q}_{m}\right\|_{N_{2}}=\boldsymbol{q}_{m}^{\dagger} M_{2}^{\dagger} M_{2} \boldsymbol{q}_{m}
$$


and $P$ be a discretization of the propagator $\exp \left(T L_{m}\right)$. In discrete form, $(15)$ can be rewritten as

$$
\begin{aligned}
\boldsymbol{q}_{m a x}^{T, m} & =\arg \max \frac{\boldsymbol{q}_{m}^{\dagger} P^{\dagger} M_{1}^{\dagger} M_{1} P \boldsymbol{p}_{m}}{\boldsymbol{q}_{m}^{\dagger} M_{2}^{\dagger} M_{2} \boldsymbol{q}_{m}} \\
& =\arg \max \frac{\boldsymbol{p}_{m}^{\dagger} M_{2}^{-1^{\dagger}} P^{\dagger} M_{1}^{\dagger} M_{1} P M_{2}^{-1} \boldsymbol{p}_{m}}{\boldsymbol{p}_{m}^{\dagger} \boldsymbol{p}_{m}}
\end{aligned}
$$

since $N_{2}$ is a norm. The optimal perturbation and gain can therefore be computed as the leading eigenvector and eigenvalue of

$$
\mathcal{T}=M_{2}^{-1^{\dagger}} P^{\dagger} M_{1}^{\dagger} M_{1} P M_{2}^{-1} .
$$

$M_{1}$ and $M_{2}$ are block-diagonal operators with $5 \times 5$ blocks. Consequently, applying $M_{2}^{-1}$ to a vector is straightforward. The action of $\mathcal{T}$ is computed in a matrix-free framework ${ }^{14}$ and coupled with the KrylovSchur solver in SLEPc (See Hernandez et al. ${ }^{20}$ for details)

RESUlts For azimuthal wave numbers $m=0,1,2,3$, optimal perturbations were computed for different time horizons $1 \leq T \leq 50$. In nearly all cases (with the exception of $m=0$ and $T=50$ discussed later), the optimal perturbation takes the form of a vortical wave packet localized inside the duct near the nozzle exit. This wave packet experiences transient growth as it travels downstream. This convection is visualized in figure 9 in terms of the contribution to the norm of a position $x$,

$$
N(x) \equiv \int_{0}^{\infty}\left(\rho_{b}\left|\boldsymbol{u}_{m}\right|^{2}+\frac{p_{b}}{\rho_{b}}\left|\rho_{m}\right|^{2}+\frac{\rho_{b}^{3}}{\gamma^{2}(\gamma-1) \mathrm{Ma}^{4} p_{b}}\left|T_{m}\right|^{2}\right) r \mathrm{~d} r .
$$

The convection velocity is found to be near half the center-line velocity, and it decreases as the wave packet moves downstream. Figure 10 shows the velocity perturbation $u_{x}$ for $m=1$ and $T=40$ (most amplified case) at $t=0$ and 40 . In figure 11, the time evolution of the norm of optimal perturbations is represented in dashed lines for each of the optimal initial conditions. The solid line represents the envelop function $G_{m}(T)$ as a function of the time horizon.

Axisymmetric perturbations (figure 11a) display a different behavior than cases with $m \neq 0$. For $m=0$, perturbations grow fast over a time interval of the order of the convection time across the potential core, as shown in figure 11a. They then decay quickly, much faster than the jet least damped eigenmode, which is characteristic of a non-modal behavior. For $m \neq 0$, perturbations decay much slower after reaching their maximum amplitude. The highest gain $G_{m}(T)$ is achieved for $m=1$ and $T=40$, and this maximum occurs for $t \approx 35$. At this instant, the wave-packet is far downstream of the potential core. This observation is consistent with local findings, i.e. Michalke, ${ }^{25}$ that helical modes may be locally unstable even for bell-shaped velocity profiles.

For all time horizons, the largest gains are observed for $m=1$, followed by $m=2$ and $m=3$. Axisymmetric perturbations are always the least amplified in our calculations. This dominance of helical perturbations suggests the importance of the lift-up mechanism.

The nature of the optimal perturbation for $m=0$ and $T=50$ is totally different: it consists of an incoming acoustic wave, which reaches the nozzle exit around $t=40$ and gives rise to vortical perturbations in the shear layer, which are amplified as they travel downstream.

\section{Conclusion}

The effects of non-parallelism on the linear global stability of subsonic jets have been considered. Axisymmetric global modes provide some understanding of the nature of the preferred mode. It cannot be understood as the least damped eigenmode as the present results show that as far as shear layer modes are considered, the least damped modes are localized far downstream of the potential core, i.e in a region where coherent structures are not observed. However, as the frequency of the modes increases, vortical structure start to be present in the potential core. Although additional results are needed, this suggests that the preferred mode could be understood by considering both the mode decay rate and its streamwise localization.

The non-normal study has shown that perturbations with an azimuthal wave number $m=1$ exhibit the largest transient growth. These perturbations are fully three dimensional, and different transient growth 

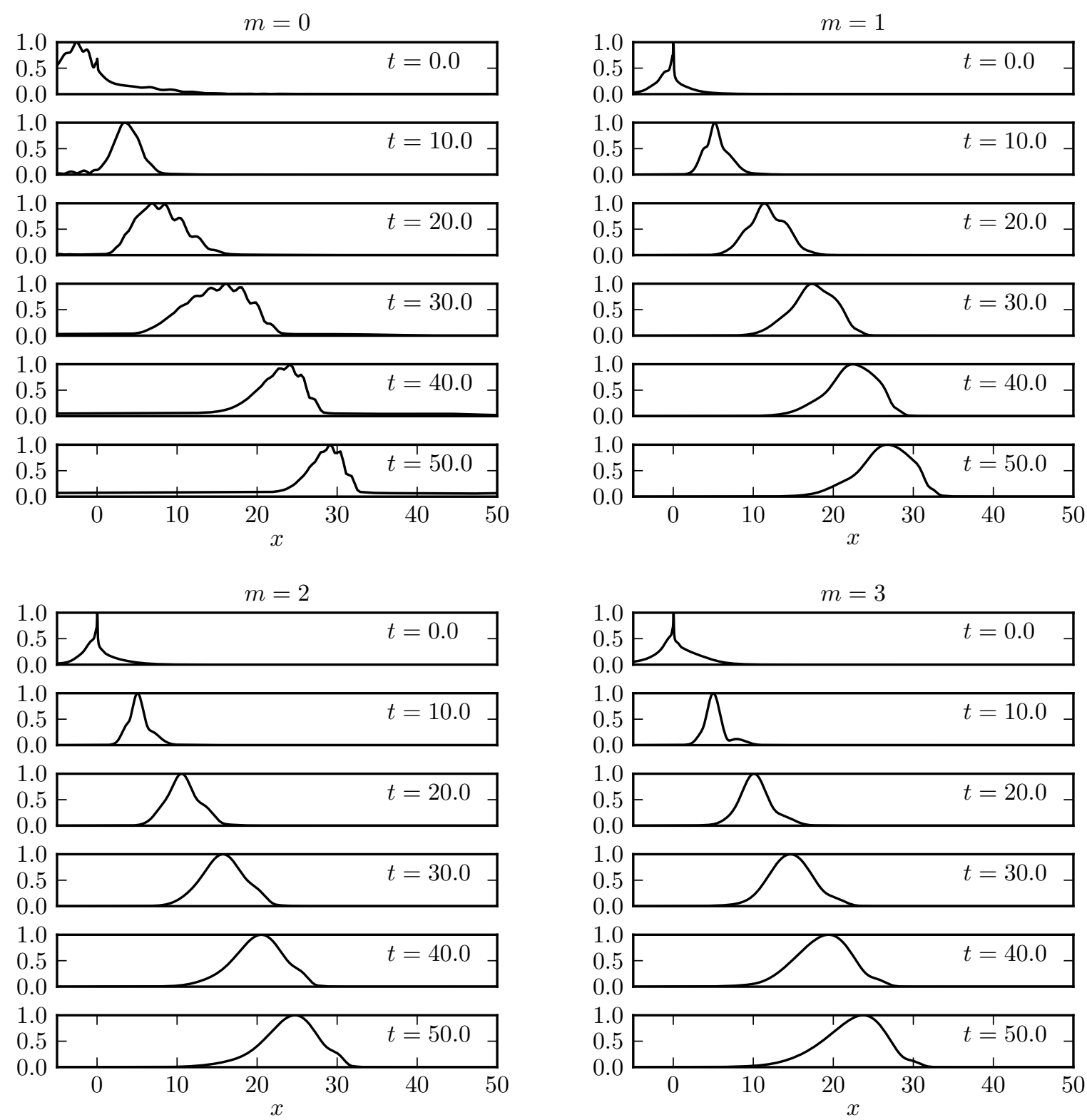

Figure 9. Axial distribution of the wave-packet for the optimal perturbation at $T=20$ for azimuthal wave-number $m=0,1,2,3 . \frac{N(x)}{\max N}$ is represented as a function of the axial position at different times, as indicated in the figures.

mechanisms are at play. Whereas axisymmetric perturbations reach their maximum amplitudes downstream within the potential core, transient mechanisms are still active in the self similar region for $m \neq 0$. Results also show that acoustic perturbation can effectively excite shear layer perturbations.

\section{Acknowledgments}

The authors wish to thank M. Fosas de Pando and C. Arratia for their comments. This work was supported by DGA grant number 2009.60.034.00.470.75.01 and was performed using HPC resources from GENCI-CINES (Grant 2011-026451). 


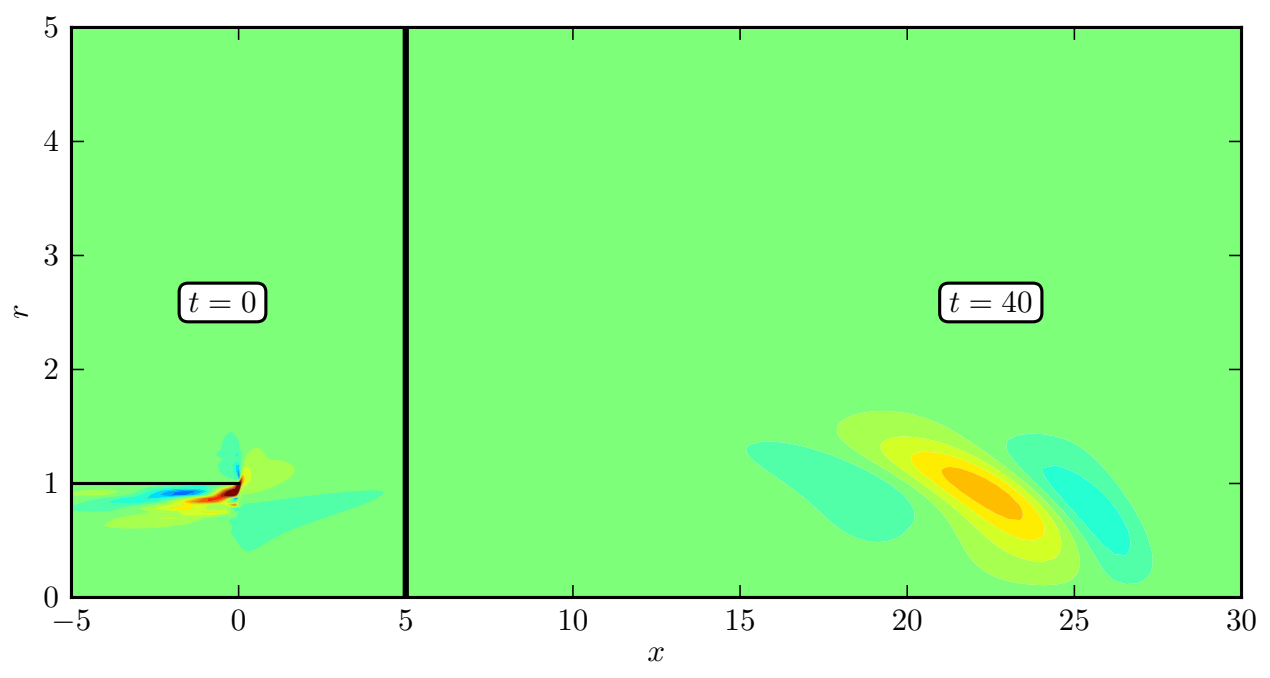

Figure 10. Axial velocity distribution of the initial condition and of the corresponding optimal response for $m=1$ and $T=40$. Initial and final conditions are respectively drawn in the left and right part of the figure with different color scales. 

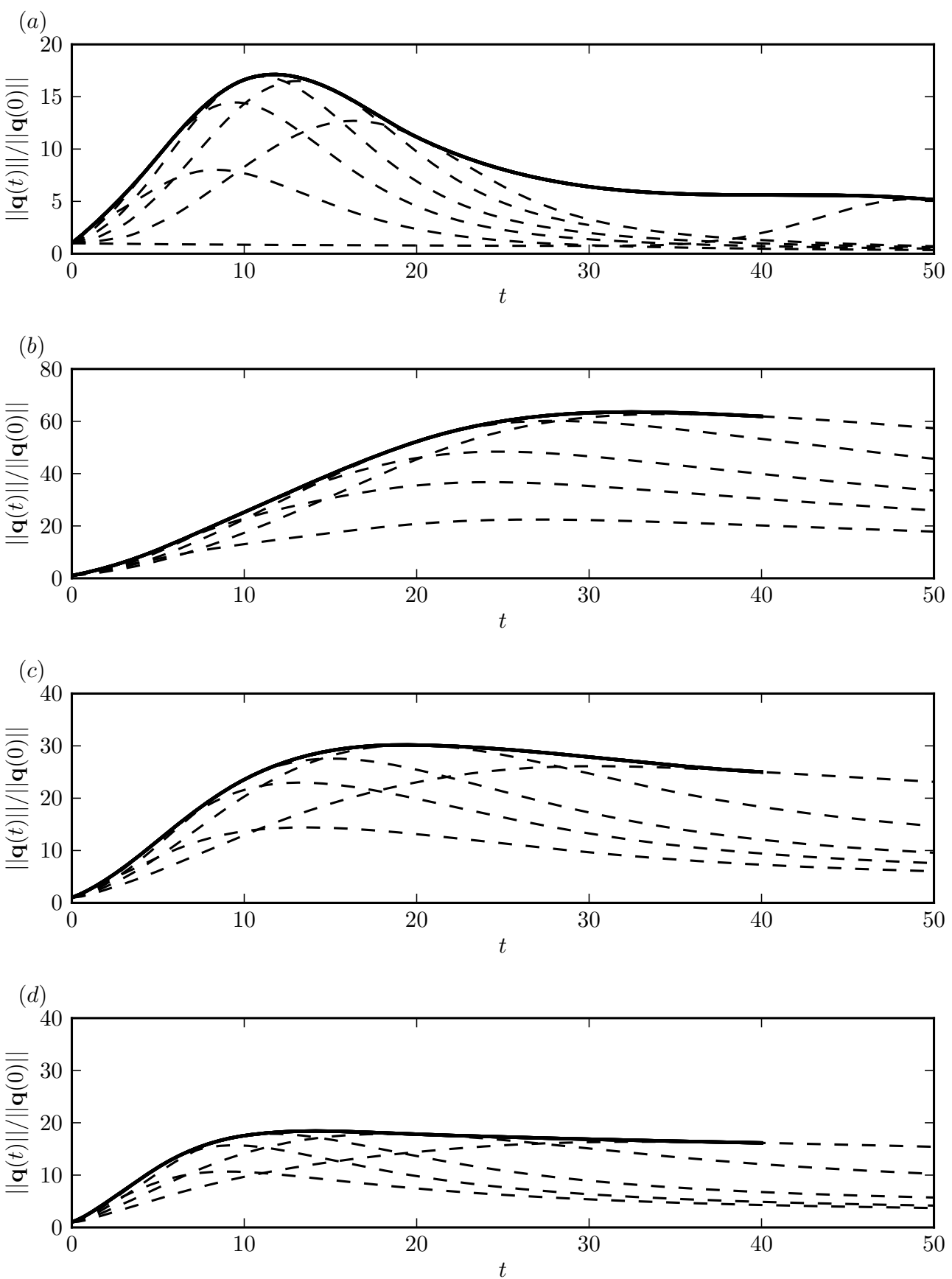

Figure 11. Optimal perturbations for different azimuthal modes $((a): m=0,(b): m=1,(c): m=2,(d): m=3)$. The envelope, represented by the bold line, corresponds to the optimal gain $G(t)$. Dashed lines correspond to the evolution of the norm for initial conditions optimized for time $T=1,5,10,20$ and 40 . 


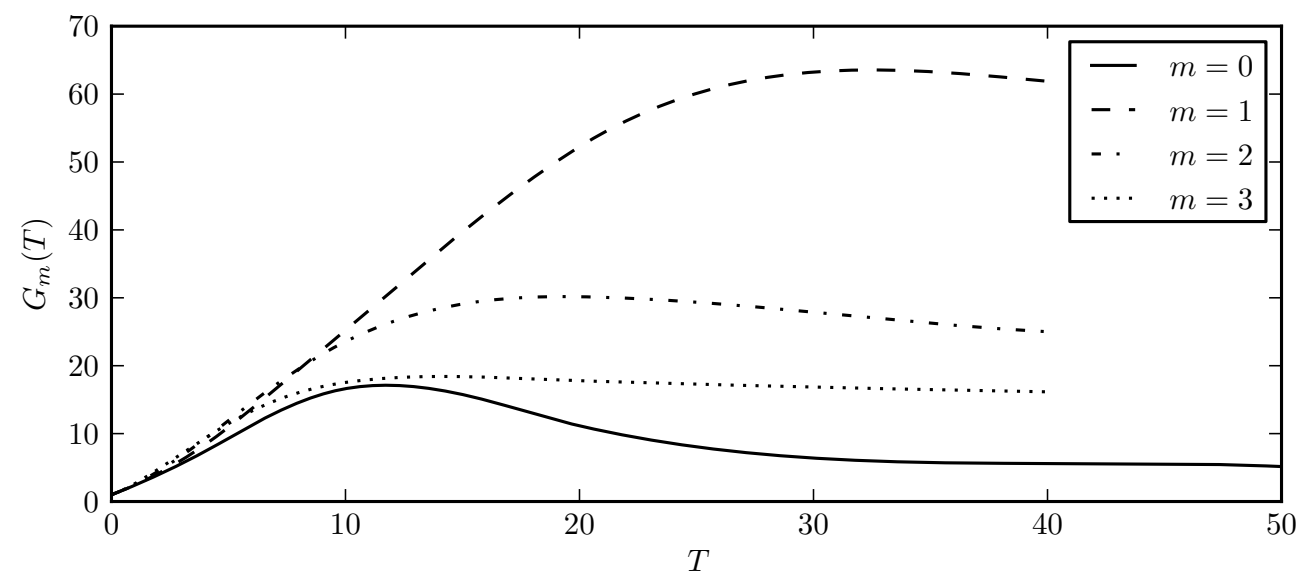

Figure 12. Envelope of maximal transient growth for different angular modes.

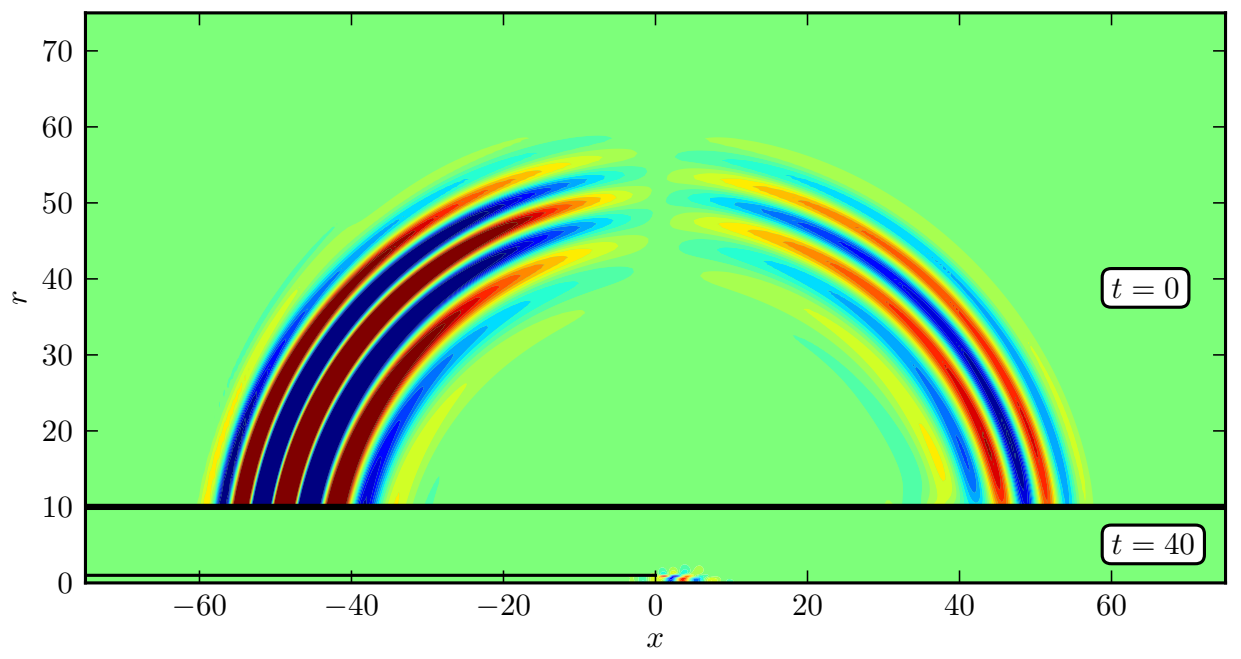

Figure 13. Axial velocity distribution of the initial condition and of the corresponding optimal response for $m=0$ and $T=50$. Initial and final conditions are respectively drawn in the top and bottom part of the figure with different color scales. 


\section{References}

${ }^{1}$ E. Akervik, L. Brandt, D.S. Henningson, J. Hoepffner, O. Marxen, and P. Schlatter. Steady solutions of the Navier-Stokes equations by selective frequency damping. Phys. Fluids, 18(6):068102, 2006.

${ }^{2}$ E. Akervik, U. Ehrenstein, F. Gallaire, and D.S. Henningson. Global two-dimensional stability measures of the flat plate boundary-layer flow. Eur. J. Mech. B-Fluid, 27(5):501-513, 2008.

${ }^{3}$ P.R. Amestoy, I.S. Duff, and J.Y. L'Excellent. Multifrontal parallel distributed symmetric and unsymmetric solvers. Comput. Method. Appl. M., 184(2-4):501-520, 2000.

${ }^{4}$ S. Balay, K. Buschelman, V. Eijkhout, W.D. Gropp, D. Kaushik, M.G. Knepley, L. Curfman McInnes, B.F. Smith, and H. Zhang. PETSc users manual. Technical Report ANL-95/11 - Revision 3.0.0, Argonne National Laboratory, 2008. Available at http://www.mcs.anl.gov/petsc/petsc-as/.

${ }^{5}$ G. K. Batchelor and A. E. Gill. Analysis of the stability of axisymmetric jets. J. Fluid Mech., 14(04):529, 1962.

${ }^{6} \mathrm{~J}$. Berland, C. Bogey, O. Marsden, and C. Bailly. High-order, low dispersive and low dissipative explicit schemes for multiple-scale and boundary problems. J. Comput. Phys., 224(2):637-662, 2007.

${ }^{7}$ H. M. Blackburn, D. Barkley, and S. J. Sherwin. Convective instability and transient growth in flow over a backward-facing step. J. Fluid Mech., 603, April 2008.

${ }^{8}$ C. Bogey and C. Bailly. Three-dimensional non-reflective boundary conditions for acoustic simulations: far field formulation and validation test cases. Acta Acust., 88:463-471, 2002.

${ }^{9} \mathrm{C}$. Bogey and C. Bailly. Computation of a high reynolds number jet and its radiated noise using large eddy simulation based on explicit filtering. Comput. Fluids, 35(10):1344-1358, 2006.

${ }^{10}$ L.C. Cheung and S.K. Lele. Linear and nonlinear processes in two-dimensional mixing layer dynamics and sound radiation. J. Fluid Mech., 625:321, April 2009.

${ }^{11}$ D.G. Crighton and M. Gaster. Stability of slowly diverging jet flow. J. Fluid Mech., 77(02):397, 1976.

12 J.D. Crouch, A. Garbaruk, and D. Magidov. Predicting the onset of flow unsteadiness based on global instability. J. Comput. Phys., 224(2):924-940, June 2007.

${ }^{13}$ S.C. Crow and F.H. Champagne. Orderly structure in jet turbulence. J. Fluid Mech., 48(03):547, 1971.

${ }^{14}$ M. Fosas de Pando, D. Sipp, and P.J. Schmid. Matrix extraction technique for global stability of compressible flows: application to mixing and boundary layers. AIAA paper (unpublished), 2011.

${ }^{15}$ W.S. Edwards, L.S. Tuckerman, R.A. Friesner, and D.C. Sorensen. Krylov methods for the incompressible Navier-Stokes equations. J. Comput. Phys., 110(1):82-102, 1994.

${ }^{16}$ J.B. Freund. Noise sources in a low-Reynolds-number turbulent jet at mach 0.9. J. Fluid Mech., 438:277-305, 2001.

${ }^{17}$ X. Garnaud, L. Lesshafft, P. Schmid, and J.-M. Chomaz. A relaxation method for large eigenvalue problems, with an application to flow stability analysis. submitted to Journal of Computational Physics, 2011.

${ }^{18} \mathrm{~K}$. Gudmundsson and T. Colonius. Parabolized stability equation models for turbulent jets and their radiated sound. AIAA paper 2009-3380, 2009.

${ }^{19}$ A. Hanifi, P.J. Schmid, and D.S. Henningson. Transient growth in compressible boundary layer flow. Phys. Fluids, $8(3): 826-837,1996$.

${ }^{20}$ V. Hernandez, J.E. Roman, and V. Vidal. SLEPc: a scalable and flexible toolkit for the solution of eigenvalue problems. ACM T. on Math. Software, 31(3):351362, September 2005.

${ }^{21}$ R.B. Lehoucq and A.G. Salinger. Large-Scale eigenvalue calculations for stability analysis of steady flows on massively parallel computers. Inter. J. Numer. Meth. Fl., 36:309-327, 1999.

${ }^{22}$ C.J. Mack and P.J. Schmid. A preconditioned krylov technique for global hydrodynamic stability analysis of large-scale compressible flows. J. Comput. Phys., 229(3):541-560, 2010.

${ }^{23}$ O. Marquet, D. Sipp, and L. Jacquin. Sensitivity analysis and passive control of cylinder flow. J. Fluid Mech., 615:221-252, 2008.

${ }^{24}$ P. Meliga, D. Sipp, and J.-M. Chomaz. Effect of compressibility on the global stability of axisymmetric wake flows. J. Fluid Mech., 660:499-526, August 2010.

${ }^{25}$ A. Michalke. Survey on jet instability theory. Prog. Aerosp. Sci., 21:159-199, 1984.

${ }^{26}$ P.A. Monkewitz and K. Sohn. Absolute instability in hot jets. AIAA J., 26(8):911-916, August 1988.

${ }^{27}$ A. Monokrousos, E. Akervik, L. Brandt, and D.S. Henningson. Global three-dimensional optimal disturbances in the blasius boundary-layer flow using time-steppers. J. Fluid Mech., 650:181, March 2010.

${ }^{28}$ J.W. Nichols and S.K. Lele. Global modes and transient response of a cold supersonic jet. J. Fluid Mech., 669:225-241, January 2011.

${ }^{29}$ P. Plaschko. Helical instabilities of slowly divergent jets. J. Fluid Mech., 92(02):209, 1979.

${ }^{30}$ S.C. Reddy and D.S. Henningson. Energy growth in viscous channel flows. J. Fluid Mech., 252(-1):209-238, 1993.

${ }^{31}$ Y. Saad. Iterative methods for sparse linear systems, second edition. SIAM, 2003.

${ }^{32}$ R.D. Sandberg. Governing equations for a new compressible Navier-Stokes solver in general cylindrical coordinates. Technical Report AFM-07/07, University of Southampton, 2007. Available at http://eprints.soton.ac.uk/49523/.

${ }^{33}$ H. Schlichting. Boundary-layer theory. McGraw-Hill, New York, 1955.

${ }^{34}$ P.J. Schmid and D.S. Henningson. Stability and Transition in Shear Flows. Springer, 2001.

${ }^{35}$ K. Sohn. Absolute instability in hot jets. M.S. thesis, UCLA, 1986. 